\title{
Fatores associados ao excesso de peso e baixa estatura em escolares nascidos com baixo peso
}

\author{
Factors associated with excess weight and stunting \\ in schoolchildren born with low birth weight
}

Renata Cavalcante Kuhn-Santos ${ }^{1}$

Fabíola Isabel Suano-Souza ${ }^{1}$

Rosana Fiorini Puccini ${ }^{1}$

Maria Wany Louzada Strufaldi ${ }^{1}$

${ }^{1}$ Departamento de Pediatria, Escola Paulista de Medicina, Universidade Federal de São Paulo. R. Botucatu 598, Vila Clementino. 04023-062 São Paulo SP Brasil. renata_kuhn@yahoo.com.br

\begin{abstract}
The scope of this study is to assess the nutritional status of low birth weight (LBW) children and the possible associations with independent maternal variables, gender and neonatal history. It involved a cross-sectional study with 544 $L B W$ schoolchildren (five to ten years of age) in the metropolitan area of São Paulo. Variables: the neonatal data of liveborn infant declarations and the current weight and height of the mothers were collected. The weight and stature used to calculate the height/age $z$ (HAZ) score and the body mass index (BMI) of children were evaluated. Among the LBW children $6.2 \%$ were of short stature, $12.3 \%$ overweight and $8.6 \%$ obese. There was an association between short stature in LBW schoolchildren and short maternal stature $<150 \mathrm{~cm}$ $(O R=6.94 ; 95 \%$ CI 2.34-20.6). Excess weight) obesity in LBW children was independently associated with overweight/obesity of the mother (OR $=2.40 ; 95 \%$ CI 1.44-4.01), and the male gender $(\mathrm{OR}=1.77 ; 95 \%$ CI 1.06-2.95). A fifth of schoolchildren with low birth weight were overweight, which was associated with current maternal nutritional status and the male gender and stunting was associated with maternal stature.
\end{abstract}

Key words Low birth weight, Stunting, Children, Excess weight, Obesity
Resumo O objetivo deste artigo é avaliar a condição nutricional de crianças com baixo peso ao nascer (BPN) e possíveis associações com variáveis independentes maternas, sexo e antecedentes neonatais Estudo transversal com 544 escolares com BPN (5 a 10 anos de idade) da região metropolitana de São Paulo. Variáveis: dados neonatais das declarações de nascidos vivos (peso ao nascere idade gestacional), informações sobre a gestação $e$ a condição nutricional atual das mães. A avaliação da condição nutricional dos escolares foi realizada por meio da obtenção dos dados de peso e estatura utilizados cálculo do escore $z$ da estatural idade (ZEI) e indice de massa corporal (ZIMC). Observou-se baixa estatura; sobrepeso e obesidade em 6,2\%, 8,6\% e 12,3\% das crianças avaliadas, respectivamente. A presença de baixa estatura nos escolares associou-se com estatura materna $<150$ $\mathrm{cm}(\mathrm{OR}=6,94$; IC95\% 2,34-20,6). O sobrepeso/ obesidade nas crianças com BPN associou-se de forma independente com o sobrepeso/obesidade da mãe $(O R=2,40 ;$ IC95\% 1,44-4,01) e o sexo masculino $(O R=1,77 ;$ IC95\% 1,06-2,95). Um quinto dos escolares com BPN apresentaram excesso de peso, que se associou à condição nutricional materna atual e ao gênero masculino; a baixa estatura associou-se à estatura materna.

Palavras-chave Recém-nascido de baixo peso, Nanismo, Criança, Sobrepeso, Obesidade 


\section{Introdução}

O baixo peso ao nascer (BPN) é definido como peso de nascimento inferior a 2500 gramas. Os fatores de risco para a sua ocorrência relacionam-se a condições adversas fetais (alterações genéticas, malformações) e maternas (diabetes, hipertensão, infecção, desnutrição, tabagismo). Pode haver associação com prematuridade e/ou crescimento intrauterino restrito (CIUR) ${ }^{1,2}$.

As taxas de BPN variam nas diferentes regiões no mundo, encontrando patamares próximos de $15 \%$ em países em desenvolvimento, o dobro do observado em países desenvolvidos (7\%). No Brasil as taxas de BPN apresentam comportamento paradoxal, com diferenças entre as regiões e entre grupos populacionais procedentes de diferentes classes sociais ${ }^{3}$. Baseado em informações sobre nascidos vivos, a taxa de BPN no ano de 2011 foi de 8,5\% no país, com valores semelhantes observados, em 2013, no Estado de São Paulo $(9,1 \%)$, na Região Metropolitana $(9,4 \%)$ e no município de São Paulo $(9,6 \%)^{4}$.

A incidência de BPN, utilizada como um indicador de saúde pública, está relacionada de forma direta com a morbidade infantil, associa-se com inadequação do crescimento pôndero-estatural, prejuízo do desenvolvimento neuropsicomotor e com maior risco para doenças crônicas não transmissíveis como obesidade, hipertensão arterial sistêmica, diabetes mellitus tipo 2 e doenças cardiovasculares no futuro ${ }^{5,6}$.

Há associação inversa entre o peso ao nascer e a frequência de déficit de estatura na infância e vida adulta, assim como maior chance de baixa estatura entre os nascidos pequenos para idade gestacional (PIG) ${ }^{7,8}$. O crescimento pós-natal é influenciado por fatores ambientais, práticas alimentares e pelo potencial genético refletido pela estatura parental ${ }^{9,10}$. Nesse contexto, espera-se que a criança com BPN faça recuperação nutricional precoce (catch-up growth) para entrar no canal de crescimento considerado adequado para a idade ${ }^{11,12}$. Sabe-se que a recuperação lenta se associa com comprometimento estatural e pior desenvolvimento cognitivo, e que, por sua vez, o ganho de peso excessivo, especialmente nos primeiros anos, associa-se com risco aumentado da obesidade na infância e adolescência; dislipidemias, diabetes mellitus, hipertensão arterial e doenças cardiovasculares na vida adulta ${ }^{13}$.

A trajetória de crescimento "ideal", que levaria a recuperação nutricional sem aumentar o risco de doenças crônicas para crianças com $\mathrm{BPN}$, ainda não é completamente conhecida ${ }^{13}$, especialmente em países como o Brasil, onde os determinantes do BPN associam-se, principalmente, a doenças nutricionais maternas ligadas tanto a excessos (obesidade materna, ganho excessivo de peso durante a gestação) quanto a carências (anemia ferropriva, desnutrição energético proteica) ${ }^{14}$.

Dados extraídos da Pesquisa de Orçamentos Familiares (2008-2009) ${ }^{15}$ e da Pesquisa Nacional de Saúde do Escolar (PeNSe) ${ }^{16}$ mostraram que 25 a $30 \%$ dos pré-escolares e escolares brasileiros estão com excesso de peso e que a frequência de baixa estatura, apesar de ter sofrido queda importante nas últimas décadas, ainda acomete por volta de 3,0 a $12,2 \%$ das crianças menores de 5 anos, com prevalências maiores nas regiões Norte e Nordeste. Somados, excesso de peso e baixa estatura, acometem mais de $30 \%$ das crianças brasileiras.

Tendo em vista que o baixo peso ao nascer e as inadequações nutricionais na infância (excesso de peso e baixa estatura) são condições frequentes no nosso meio, e hoje representam fatores para o desenvolvimento de doenças em curto e longo prazos, resolveu-se realizar este estudo que tem como objetivo descrever a condição nutricional de crianças de 5 a 10 anos de idade da região metropolitana de São Paulo, que nasceram com baixo peso, avaliar possíveis associações das alterações na condição nutricional (sobrepeso/ obesidade) e baixa estatura com variáveis maternas, sexo e antecedentes neonatais.

\section{Método}

\section{Desenho de estudo}

Foi realizado estudo transversal, entre os anos de 2012 - 2013, com 544 escolares com idade entre 5 a 10 anos, que nasceram com baixo peso (< 2500 gramas) no município de Embu das Artes (região metropolitana de São Paulo - Brasil). O município de Embu das Artes possuía, na época da pesquisa, renda per capita de 1,7 salários mínimos, taxa de BPN de $9,8 \%$, e $89,1 \%$ dos estudantes estavam matriculados em escolas públicas ${ }^{17}$.

Para composição da casuísta foi realizado um cruzamento entre os bancos de dados das secretarias de educação (municipal e estadual) do município de Embu das Artes, com os dados da declaração de nascidos vivos (DNV) da Fundação Sistema Estadual de Análise de Dados (SEADE). Identificou-se 22.643 crianças matriculadas em escolas públicas do município e destas, 996, entre 5 a 10 anos de idade, que tiveram BPN. 
As crianças com BPN identificadas foram contatadas nas escolas que estavam matriculadas e convidadas a comparecer, com os responsáveis, às unidades básicas de saúde (UBS) mais próximas ao seu domicílio para entrevista e avaliação antropométrica. Compareceram para essa avaliação 569/996 (57,1\%) crianças com suas famílias.

Não foram incluídas no estudo os escolares com doenças crônicas (7 com encefalopatia crônica não evolutiva, 1 com Síndrome de Down), aqueles cujos responsáveis não concordaram com a avaliação antropométrica (16) e os com idade igual ou superior a 11 anos (1). Dessa forma, a casuística final foi composta por 544 crianças.

Foi realizada a análise das perdas, a partir dos dados das DNV e não houve diferença estatisticamente significante entre aqueles que compareceram e os escolares que não compareceram (n = 427) para avaliação e entrevista em relação ao gênero, idade, Apgar de $5^{\circ}$ minuto, tipo de parto, idade gestacional e peso ao nascer.

$\mathrm{O}$ presente estudo foi aprovado pelo Comitê de Ética em Pesquisa da Universidade Federal de São Paulo, pelas Secretarias da Saúde e da Educação do município de Embu das Artes e foi parte do projeto Pesquisa para o SUS Gestão Compartilhada em Saúde (PPSUS - Morbidade, crescimento e desenvolvimento de escolares de 6 a 10 anos de idade nascidos com baixo peso ao nascer - integralidade e intersetorialidade na atenção à criança no sistema local de saúde Embu SP).

\section{Dados coletados}

Declaração de nascidos vivos (DNV): informações sobre a mãe (idade da mãe, escolaridade e número de gestações), gestação (única ou múltipla e número de consultas no pré-natal), parto (idade gestacional: $<32$ semanas, 32 a 36 semanas e $\geq 37$ semanas; tipo de parto e local de nascimento - hospital ou domicílio) e recém-nascido (sexo, etnia, presença de malformação e peso ao nascer).

Condição nutricional materna: a informação sobre peso e estatura da mãe foi referida durante a entrevista e os dados foram utilizados para cálculo do índice de massa corporal (IMC), que foi classificado segundo recomendação da Organização Mundial de Saúde ${ }^{18}$.

Condição nutricional das crianças: os escolares foram pesados em balança do tipo plataforma, digital, com capacidade de até 150 quilos. A medida de peso foi tomada com a criança vestindo roupas leves, descalça, permanecendo ereta no centro da balança, com os braços esticados ao longo do corpo. A estatura foi medida utilizando-se estadiômetro portátil (milimetrado). A aferição foi efetuada com as crianças em posição ereta, descalças, calcanhares unidos e braços ao longo do corpo. O IMC foi expresso em $\mathrm{Kg} / \mathrm{m}^{2}$ e os valores calculados pelo programa WHO Anthro Plus ${ }^{\circledR}$, expressos em escore Z. Os pontos de corte utilizados foram definidos de acordo com os referenciais da OMS, 2006/2007 ${ }^{19}$.

\section{Análise Estatística}

Os dados foram digitados e consolidados em Planilha do Excel (Office Microsoft ${ }^{\circledR}$ ) e a análise estatística foi feita com o Pacote Estatístico IBM SPSS ${ }^{\circledR}$ 20.0. As variáveis contínuas foram testadas quanto a sua normalidade. As variáveis com distribuição normal foram descritas na forma de média e desvio padrão e comparadas por meio do teste de t-Student. As variáveis com distribuição não normal foram apresentadas na forma de mediana (mínimo e máximo) e comparadas por meio do teste de Mann-Whitney. As variáveis categóricas foram mostradas na forma de tabelas (valor absoluto e percentual), comparadas por meio do teste do Qui-quadrado e calculado o odds ratio (OR) com intervalo de confiança de 95\%. Para análise multivariada foi utilizada a regressão binária logística método forward LR e incluídas aquelas com $\mathrm{p}<0,10$. O nível de significância adotado foi $5 \%$.

\section{Resultados}

Na Tabela 1 estão descritas as características gerais da população estudada. Em relação às mães, a mediana de idade, na época do nascimento das crianças, foi de 25 anos; somente 202 (38,3\%) realizaram mais do que seis consultas de pré-natal, a maioria tinha filhos anteriores 303 (71,8\%), metade viviam com companheiro 279 (50,5\%) e 267 (48,7\%) tinham mais de 8 anos de escolaridade (Tabela 1). Quanto à condição nutricional materna atual, $26(11,5 \%)$ e $164(41,4 \%)$ das mães tinham estatura $<150 \mathrm{~cm}$ e excesso de peso (sobrepeso e obesidade), respectivamente (Tabela 1).

A maioria dos partos foi vaginal $314(55,4 \%)$ e à termo ( $>37$ semanas). A mediana do peso ao nascer foi 2252 gramas (variação: 620,0 a 2495 gramas), sendo que $91,2 \%$ estavam entre 1500 e 2499g (Tabela 1).

Em relação aos escolares, a mediana de idade foi 8,2 anos (variação: 5,3 a 10,9 anos) e predo- 
Tabela 1. Características gerais e condição nutricional da população estudada, Embu das Artes, 2012/2013.

\begin{tabular}{|c|c|c|}
\hline Variáveis & $\mathbf{n}$ & $\%$ \\
\hline Condição marital & 552 & \\
\hline Com companheiro & 279 & 50,5 \\
\hline Idade & 569 & \\
\hline$<20$ anos & 117 & 20,6 \\
\hline 20 a 34 anos & 375 & 65,9 \\
\hline$>34$ anos & 77 & 13,5 \\
\hline Escolaridade maternal (anos) & 550 & \\
\hline 0 a 3 & 59 & 10,7 \\
\hline 4 a 7 & 224 & 40,7 \\
\hline 8 ou mais & 267 & 48,6 \\
\hline Filhos anteriores & 427 & \\
\hline Sim & 303 & 71,0 \\
\hline Tipo de gestação & 564 & \\
\hline Única & 492 & 87,2 \\
\hline Dupla & 71 & 12,6 \\
\hline Tripla ou mais & 1 & 0,2 \\
\hline Número de visitas pré-natal & 527 & \\
\hline Nenhuma & 32 & 6,1 \\
\hline 1 a 3 & 54 & 10,2 \\
\hline 4 a 6 & 239 & 45,4 \\
\hline 7 ou mais & 202 & 38,3 \\
\hline Idade gestacional (semanas) & 550 & \\
\hline$<32$ & 44 & 8,0 \\
\hline 32 a 36 & 217 & 39,5 \\
\hline$\geq 37$ & 289 & 52,5 \\
\hline Tipo de parto & 567 & \\
\hline Vaginal & 314 & 55,4 \\
\hline Gênero & 569 & \\
\hline Feminino & 322 & 56,6 \\
\hline Etnia & 441 & \\
\hline Branca & 200 & 45,4 \\
\hline Negra & 29 & 6,6 \\
\hline Local de nascimento & 554 & \\
\hline Hospital & 544 & 98,2 \\
\hline Mal formações graves & 461 & \\
\hline Não & 456 & 98,9 \\
\hline Peso ao nascer & 569 & \\
\hline$<1000 \mathrm{~g}$ & 12 & 2,1 \\
\hline 1000 a $1500 \mathrm{~g}$ & 38 & 6,7 \\
\hline 1500 a $2500 \mathrm{~g}$ & 519 & 91,2 \\
\hline Estatura / idade (escore z) & 544 & \\
\hline Baixa estatura & 34 & 6,2 \\
\hline Índice de massa corporal (escore z) & 544 & \\
\hline Magreza & 26 & 4,8 \\
\hline Eutrófico & 404 & 74,3 \\
\hline Sobrepeso & 67 & 12,3 \\
\hline Obesidade & 47 & 8,6 \\
\hline Estatura materna & 409 & \\
\hline$<150 \mathrm{~cm}$ & 26 & 11,5 \\
\hline
\end{tabular}

continua
Tabela 1. Características gerais e condição nutricional da população estudada, Embu das Artes, 2012/2013.

\begin{tabular}{lrr}
\hline \multicolumn{1}{c}{ Variáveis } & \multicolumn{1}{c}{ n } & \multicolumn{1}{c}{$\%$} \\
\hline Condição nutricional maternal atual & 396 & \\
Desnutrição & 26 & 6,6 \\
Eutrofia & 206 & 52,0 \\
Sobrepeso & 122 & 30,8 \\
Obesidade I & 29 & 7,3 \\
Obesidade II & 13 & 3,3 \\
\hline
\end{tabular}

minou o sexo feminino 322 (56,6\%). A mediana do escore ZEI foi de $-0,33(-3,33$ a $+3,68)$ e do escore ZIMC foi de $-0,23(-3,85 \mathrm{a}+3,42)$. Os distúrbios nutricionais mais frequentes foram sobrepeso/obesidade: 114 (20,9\%) e baixa estatura: $34(6,2 \%)$ (Tabela 1$)$.

A estatura materna $<150 \mathrm{~cm}$ associouse de forma independente com presença de baixa estatura $(\mathrm{ZEI}<-2)$ nos escolares $(\mathrm{OR}=6,94$; IC $95 \%$ 2,34-20,6) (Tabela 2). Sexo masculino $(\mathrm{OR}=1,77$; IC95\%:1,06;2,95) e excesso de peso materno $(\mathrm{OR}=2,40$; IC95\%:1,44-4,01) associaram-se com maior risco de sobrepeso/obesidade nas crianças (Tabela 3). Todas as demais variáveis estudadas não mostraram associação estatisticamente com a condição nutricional dos escolares.

\section{Discussão}

O presente estudo demonstrou elevado percentual de alterações da condição nutricional em idade precoce em escolares com BPN. Um quinto das crianças já apresenta excesso de peso e a frequência de baixa estatura foi quase o dobro da observada na mesma região ${ }^{16}$.

Existem poucos estudos avaliando a condição nutricional em longo prazo de crianças que nasceram com peso entre $1500 \mathrm{~g}$ e $2500 \mathrm{~g}$. Esse é um grupo que merece atenção pela frequência, no Brasil são cerca de 150.000 recém-nascidos/ ano nessa condição, e pelo recente conhecimento de que eles também têm maior risco de evoluir com alterações do crescimento, desenvolvimento e doenças crônicas não transmissíveis no futuro ${ }^{20}$.

Não há consenso sobre a trajetória e o momento ideal de aceleração para o crescimento dessas crianças, especialmente em países como o Brasil com elevado percentual de PIG. Em uma coorte prospectiva norte americana, com recém- 
Tabela 2. Fatores associados a baixa estatura em crianças com baixo peso ao nascer, Embu das Artes, 2012/2013.

\begin{tabular}{|c|c|c|c|c|c|}
\hline Variáveis & $\begin{array}{c}\text { Baixa } \\
\text { estatura } \\
(n=34)\end{array}$ & $\begin{array}{c}\text { Estatura } \\
\text { normal } \\
(n=510)\end{array}$ & $P$ & $\begin{array}{l}\text { Odds ratio } \\
(95 \% \text { IC) }\end{array}$ & $\begin{array}{l}\text { Odds ratio } \\
\text { ajustado } \\
(95 \% \text { IC) }\end{array}$ \\
\hline Gênero $(\mathrm{n}=544)$ & & & 0,34 & $1,37(0,68 ; 2,74)$ & \\
\hline Masculino & $17(7,3 \%)$ & $215(92,7 \%)$ & & & \\
\hline Idade materna $(\mathrm{n}=544)$ & & & 0,22 & $1,42(0,56 ; 3,56)^{1}$ & \\
\hline$<20$ anos & $3(2,7 \%)$ & $107(97,3 \%)$ & & & \\
\hline 20 a 35 anos & $25(7,0 \%)$ & $334(93,0 \%)$ & & & \\
\hline$>35$ anos & $6(8,0 \%)$ & $69(92,0 \%)$ & & & \\
\hline Situação marital ( $\mathrm{n}=527)$ & & & 0,38 & $0,70(0,35 ; 1,43)$ & \\
\hline Sem companheiro & $14(5,4 \%)$ & $245(94,6 \%)$ & & & \\
\hline Escolaridade materna $(\mathrm{n}=523)$ & & & 0,58 & $1,36(0,50 ; 3,66)$ & \\
\hline$<4$ anos & $5(8,3 \%)$ & $55(91,7 \%)$ & & & \\
\hline Filhos anteriores $(n=416)$ & & & 0,28 & $0,51(0,19 ; 1,39)$ & \\
\hline Sim & $22(7,5 \%)$ & $270(92,5 \%)$ & & & \\
\hline Idade gestacional $(\mathrm{n}=525)$ & & & 0,58 & $1,35(0,65 ; 2,77)^{2}$ & \\
\hline$\leq 31$ semanas & $3(7,7 \%)$ & $36(92,3 \%)$ & & & \\
\hline 32 a 36 semanas & $10(4,9 \%)$ & $194(95,1 \%)$ & & & \\
\hline$>37$ semanas & $20(7,1 \%)$ & $262(92,9 \%)$ & & & \\
\hline Tipo de parto $(\mathrm{n}=542)$ & & & 0,18 & $1,60(0,79 ; 3,23)$ & \\
\hline Cesariana & $19(7,8 \%)$ & $224(92,2 \%)$ & & & \\
\hline Peso ao nascer $(\mathrm{n}=544)$ & & & 0,43 & $1,92(0,70 ; 5,22)^{3}$ & \\
\hline$<1000 \mathrm{~g}$ & $1(11,1 \%)$ & $8(88,8 \%)$ & & & \\
\hline $1000-1500 \mathrm{~g}$ & $4(10,5 \%)$ & $34(89,5)$ & & & \\
\hline $1500-2500 \mathrm{~g}$ & $29(5,8 \%)$ & $468(94,2 \%)$ & & & \\
\hline ZIMC $(\mathrm{n}=544)$ & & & 0,15 & & \\
\hline Magreza & $4(15,4 \%)$ & $22(84,6 \%)$ & & $2,64(0,84 ; 2,24)^{4}$ & \\
\hline Eutrofia & $26(6,4 \%)$ & $378(93,6 \%)$ & & & \\
\hline Sobrepeso & $2(3,0 \%)$ & $65(97,0 \%)$ & & $0,52(0,18 ; 1,54)^{5}$ & \\
\hline Obesidade & $2(4,3 \%)$ & $45(95,7 \%)$ & & & \\
\hline $\begin{array}{l}\text { Condição nutricional materna } \\
(\mathrm{n}=396)\end{array}$ & & & 0,03 & & \\
\hline Desnutrição & $0(0 \%)$ & $26(100 \%)$ & & OR não calculado ${ }^{6}$ & \\
\hline Eutrofia & $20(9,7 \%)$ & $186(90,3 \%)$ & & & \\
\hline Sobrepeso & $3(2,5 \%)$ & $119(97,5 \%)$ & & $0,29(0,10 ; 0,79)^{7}$ & $0,25^{8}(0,09 ; 0,70)$ \\
\hline Obesidade & $2(4,8 \%)$ & $40(95,2 \%)$ & & & \\
\hline Estatura materna $(\mathrm{n}=409)$ & & & 0,01 & $4,9(1,79 ; 13,50)$ & $6,94^{8}(2,34 ; 20,6)$ \\
\hline$<150 \mathrm{~cm}$ & $22(5,7 \%)$ & $361(94,3 \%)$ & & & \\
\hline$\geq 150 \mathrm{~cm}$ & $6(23,1 \%)$ & $20(76,9 \%)$ & & & \\
\hline $\begin{array}{l}\text { Legenda: IMC - índice de massa corpo } \\
\text { years +<20 years; }{ }^{2} \text { OR calculado: }>37 \\
1500 \text { - } 2500 \text { g; }{ }^{4} \text { OR calculado: magreza } \\
6 \text { OR calculado: desnutrição vs, eutrofia } \\
\text { para condição nutricional materna). }\end{array}$ & $\begin{array}{l}\text { al; OR - odds } \\
\text { semanas vs, } 3 \\
\text { vs eutrofia; }{ }^{5} \mathrm{C} \\
{ }^{7} \text { OR calculad }\end{array}$ & $\begin{array}{l}\text { tio; IC - interva } \\
36 \text { semanas }+\leq \\
\text { calculado: sobr } \\
\text { obrepeso }+ \text { obe }\end{array}$ & con & $\begin{array}{l}\text { a }{ }^{1} \text { OR calculado: idade } \\
{ }^{3} \text { OR calculado: < } 1000 \text { g } \\
\text { dade vs eutrofia; } \\
\text { ofia; }{ }^{8} \text { OR da análise mul }\end{array}$ & $\begin{array}{l}+35 \text { anos vs, } 25-35 \\
+1000-1500 \mathrm{~g} \text { vs, } \\
\text { tivariada (ajustado }\end{array}$ \\
\hline
\end{tabular}




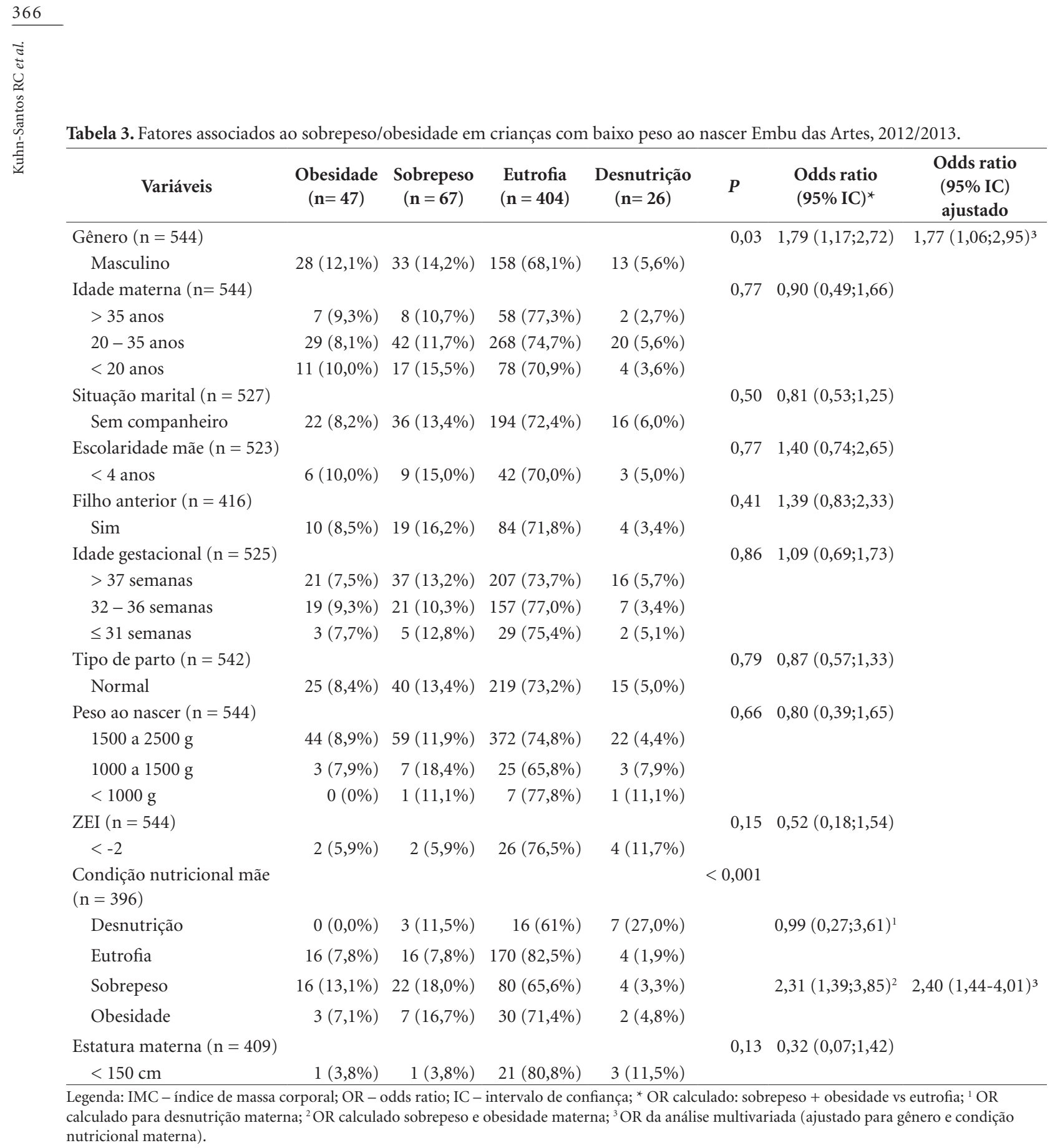

tado de evoluir com sobrepeso, principalmente quando as mães eram hipertensas e os recémnascidos pequenos para idade gestacional. $\mathrm{O}$ estudo conclui que, em nosso meio, ser pequeno para idade gestacional e ter inadequado crescimento no primeiro ano de vida são fatores de risco para distúrbios no crescimento aos dois anos de idade corrigida. Em nossa pesquisa, embora não seja possível precisar a faixa etária do catch -up, observou-se uma aceleração no crescimento e no ganho ponderal precocemente, pois já aos cinco anos de idade, muitos escolares com BPN já apresentavam excesso de peso.

Dentre os fatores de risco para desenvolvimento de adiposidade futura destacam-se: o ambiente intrauterino, a velocidade de ganho de peso nos primeiros meses/anos de vida e a presença de sobrepeso/obesidade na infância ${ }^{22}$. Estudo de coorte americana com pré-escolares mostrou que o excesso de peso aos cinco anos aumentou em sete vezes a chance de sobrepeso/ obesidade aos 14 anos de idade ${ }^{23}$. 
Além disso, a obesidade nos pais se relaciona com maior risco de obesidade nos filhos e esse fato se dá por fatores genéticos, estilo de vida (alimentação inadequada e sedentarismo) e fatores intrauterinos, como por exemplo, o excesso de ganho de peso durante a gestação ${ }^{5}$. Com base nos achados deste estudo, aproximadamente $40 \%$ das mulheres apresentavam sobrepeso/obesidade no momento da avaliação e o sobrepeso/ obesidade nas crianças com BPN associou-se de forma independente com o excesso de peso das mães. Dessa forma identificamos que crianças que tiveram BPN e têm mães obesas possuem uma somatória de fatores de risco para excesso de adiposidade e, possivelmente, para as morbidades a ela associadas. A vigilância cuidadosa da condição nutricional da mulher é uma estratégia central para o adequado crescimento da criança em curto e longo prazos $^{14}$.

De forma consonante com o que tem sido descrito nos países em desenvolvimento ${ }^{7,8}$, este estudo demonstrou que a maioria das crianças com BPN nasceu a termo. Os principais fatores de risco para BPN em nosso meio são o baixo nível socioeconômico, baixa escolaridade, falta de acesso aos serviços de saúde, tabagismo e doenças maternas como a desnutrição, baixa estatura materna, anemia, hipertensão arterial sistêmica (HAS) e diabetes mellitus ${ }^{18}$. Com relação às variáveis maternas encontradas, como escolaridade, percentual de gestações na adolescência e média de filhos e condição nutricional, nossos resultados foram semelhantes aos observados em inquéritos populacionais brasileiros ${ }^{24,25}$.

Em recente publicação sobre evolução e prevalência do BPN na população brasileira entre 1996 e 2011, foram analisados mais de $11 \mathrm{mi}$ lhões de nascimentos nas cinco regiões do país. Os autores observaram que as taxas de BPN mantiveram-se estáveis em torno de $8 \%$. A melhoria na escolaridade materna e na qualidade do pré-natal (número de visitas) reduziu o risco de BPN, especialmente nas regiões Sul e Sudeste. Entretanto, esses fatores não foram suficientes para melhorar esse indicador em grupos mais vulneráveis ${ }^{25}$.

A frequência de baixa estatura em escolares com BPN $(6,2 \%)$ foi mais elevada em comparação a um grupo de crianças com peso ao nascer adequado de mesma idade, município e condições socioeconômicas $(3,2 \%)^{17}$. Essa diferença aponta para a influência do crescimento intrauterino no crescimento pós-natal. Stein et al. ${ }^{10}$, descreveu que adultos que foram prematuros e PIG apresentam, respectivamente $1,11 \mathrm{~cm}$ e 2,35 cm de altura a menos em comparação àqueles que nasceram a termo e com peso adequado; os autores também observaram associação positiva entre o peso e estatura aos 12 meses, 24 meses e na idade pré-escolar com a estatura na vida adulta.

Entre os fatores avaliados em nossa pesquisa, a baixa estatura nas crianças com BPN associouse com a baixa estatura materna. Esse achado é semelhante a um estudo nacional que identificou a estatura materna como variável associada à estatura de crianças com oito anos de idade ${ }^{14}$.

Em uma coorte envolvendo cinco países, inclusive o Brasil, Addo et al (2013), analisaram a estatura materna e o crescimento da criança em quatro períodos da vida. Houve associação entre mulheres baixas $(<150.1 \mathrm{~cm})$ e maior chance dos filhos apresentarem baixa estatura aos dois anos de idade e na vida adulta ${ }^{25}$. A altura da mãe pode ser considerada como um indicador de fatores genéticos (determinando peso e estatura), assim como de fatores ambientais (tamanho do útero, nutrição pré-natal e dieta da criança) e é reconhecida como uma influência importante na estatura das crianças ${ }^{26,27}$.

Em nosso estudo não foram observadas diferenças quanto à frequência de baixa estatura e sobrepeso/obesidade nas diferentes faixas de peso de nascimento, de idade gestacional e entre PIG e AIG; esses resultados nos fazem considerar que mesmo as crianças menores ao nascer e/ou com menor idade gestacional, provavelmente apresentaram um catch-up mais acelerado de peso nos primeiros anos de vida e, portanto, podem estar mais susceptíveis às complicações metabólicas posteriores como relatado na literatura ${ }^{28}$.

Neste trabalho, os meninos com BPN tiveram duas vezes mais chance de apresentar sobrepeso/ obesidade em comparação com as meninas. Nos últimos anos diversos estudos têm destacado as diferenças quanto ao sexo no crescimento intrauterino e, consequentemente, no risco de BPN e desfechos futuros a ele relacionados ${ }^{29,30}$. É descrito que o dismorfismo sexual influencia no metabolismo energético, na transferência de nutrientes pela placenta, bem como nas concentrações de leptina ${ }^{30,31}$.

Eriksson et al..$^{32,33}$ demonstraram que meninos com BPN e obesidade na infância têm maior risco de desenvolver doenças cardiovasculares (DCV) na vida adulta, o que comprova um comportamento distinto entre os sexos durante a programação metabólica. Coorte conduzida por Huang et al. ${ }^{34}$ estudaram a interação entre o sexo, peso ao nascer, desenvolvimento de adiposidade e fatores de risco para DCV na adolescência. Os 
autores observaram que os meninos com BPN e ganho de peso acelerado a partir dos três anos de vida apresentavam mais fatores para DCV na adolescência. Da mesma forma, o presente estudo demonstrou maior chance para as crianças do sexo masculino com BPN apresentar sobrepeso/obesidade na infância e, consequentemente, as possíveis morbidades associadas e risco para DCV na vida adulta.

Algumas limitações devem ser consideradas neste estudo como o preenchimento incompleto das DNV; o número de crianças com BPN não localizadas; a não obtenção de dados sobre alimentação e atividade física atual e avaliação da evolução pondero-estatural.

\section{Colaboradores}

RC Kuhn-Santos - Coleta de dados, análise dos resultados, confecção do manuscrito. FI SuanoSouza - Análise dos dados, construção dos resultados, confecção do manuscrito. RF Puccini - Planejamento do estudo, análise dos resultados e confecção do manuscrito. MWL Strufaldi - Planejamento do estudo, coleta de dados, análise dos resultados e confecção do manuscrito.
Concluindo, o presente estudo identificou que, na idade pré-escolar e escolar, a maioria das crianças com BPN já fez recuperação da estatura e parcela significativa já apresenta sobrepeso/ obesidade, que foi mais frequente no sexo masculino. Houve associação entre a condição nutricional das crianças e a materna atual. O acompanhamento de forma integral da criança com BPN possibilita identificar precocemente outros fatores de risco para alterações no crescimento, na condição nutricional e provável desenvolvimento de doenças crônicas na vida adulta e reforça um papel relevante no cuidado e atenção à saúde da criança.

\section{Agradecimentos}

À Fundação de Apoio à Pesquisa do Estado de São Paulo, pelo apoio financeiro ao Projeto Pesquisa para o SUS Gestão Compartilhada em Saúde (PPSUS) 


\section{Referências}

1. World Health Organization (WHO). Technical Consultation, "Towards the development of a strategy for promoting optimal fetal growth", Report of a meeting (draft). Geneva: WHO; 2004.

2. World Health Organization (WHO). Low birthweight: Country, regional and global estimates. Geneva: United Nations Children's Fund, WHO; 2004.

3. Lima MC, Oliveira GS, Lyra CO, Roncalli AG, Ferreira MA.The spatial inequality of low birth weight in Brazil. Cien Saude Colet 2013; 18(8):2443-2452.

4. DATASUS [homepage na internet]. Tecnologia da Informação a serviço do SUS. Nascidos vivos São Paulo. [acessado 2016 Jun 15]. http://tabnet.datasus.gov.br

5. Instituto Brasileiro de Geografia e Estatística [homepage na internet]. Indicadores Sociodemográficos e de Saúde no Brasil 2009 [acessado 2016 Out 15]. Disponível em: https://ww2.ibge.gov.br/english/estatistica/populacao/indic_sociosaude/2009/indicsaude.pdf

6. Katz J, Lee AC, Kozuki N, Lawn JE, Cousens S, Blencowe H, Ezzati M, Bhutta ZA, Marchant T, Willey BA, Adair L, Barros F, Baqui AH, Christian P, Fawzi W, Gonzalez R, Humphrey J, Huybregts L, Kolsteren P, Mongkolchati A, Mullany LC, Ndyomugyenyi R, Nien JK, Osrin D, Roberfroid D, Sania A, Schmiegelow C, Silveira MF, Tielsch J, Vaidya A, Velaphi SC, Victora CG, Watson-Jones D, Black RE; CHERG Small-for-Gestational-Age-Preterm Birth Working Group. Mortality risk in preterm and small-for-gestational-age infants in low-income and middle-income countries: a pooled country analysis. Lancet 2013; 382(9890):417-425.

7. Black RE, Victora CG, Walker SP, Bhutta ZA, Christian P, de Onis M, Ezzati M, Grantham-McGregor S, Katz J, Martorell R, Uauy R; Maternal and Child Nutrition Study Group. Maternal and child undernutrition and overweight in low-income and middle-income countries. Lancet 2013; 382(9890):427-451.

8. Barros FC, Victora CG, Barros AJ, Santos IS, Albernaz E, Matijasevich A, Domingues MR, Sclowitz IK, Hallal PC, Silveira MF, Vaughan JP. The challenge of reducing neonatal mortality in middle-income countries: findings from three Brazilian birth cohorts in 1982, 1993 and 2004. Lancet 2005; 365(9462):847-854.

9. Veloso HJF, Silva AAM, Barbieri MA, Goldani MZ, Filho FL, Simões VMF, Batista RFL, Alves MTSSB, Bettiol $\mathrm{H}$. Secular trends in the rate of low birth weight in Brazilian State Capitals in the period 1996 to 2010. Cad Saúde Pública 2013; 29(1):91-101.

10. Stein AD, Barros FC, Bhargava SK, Hao W, Horta BL, Lee N, Kuzawa CW, Martorell R, Ramji S, Stein A, Richter L; Consortium of Health-Orientated Research in Transitioning Societies (COHORTS) investigators. Birth status, child growth, and adult outcomes in low- and middle-income countries. J Pediatr 2013; 163(6):1740-1746.

11. Bocca-Tjeertes IF, Kerstjens JM, Reijneveld SA, de Winter AF, Boss AF. Growth and predictors of growth restraint in moderately preterm children aged 0 to 4 years. Pediatrics 2011; 128(5):e1187-1194.

12. Ong KK, Ahmed ML, Emmett PM, Preece MA, Dunger DB. Association between postnatal catch-up growth and obesity in childhood: prospective cohort study. BMJ 2000; 320(7240):967-971.
13. Casey PH, Bradley RH, Whiteside-Mansell L, Barrett K, Gossett JM, Simpson PM. Evolution of obesity in a low birth weight cohort. J Perinatol 2012; 32(2):91-96.

14. Lei X, Chen Y, Ye J, Ouyang F, Jiang F, Zhang J. The optimal postnatal growth trajectory for term small for gestational age babies: a prospective cohort study. J Pediatr 2015; 166(1):54-58.

15. Brasil. Ministério da Saúde (MS). PNDS 2006: pesquisa nacional de demografia e saúde da criança e da mulher: relatório. Brasília: MS; 2008.

16. Instituto Brasileiro de Geografia e Estatística (IBGE). Pesquisa de orçamentos familiares 2008-2009: antropometria e estado nutricional de crianças, adolescentes e adultos no Brasil. Rio de Janeiro: IBGE; 2010.

17. Instituto Brasileiro de Geografia e Estatística (IBGE). Pesquisa nacional de saúde do escolar: 2015. Coleção Ibgeana. Rio de Janeiro: IBGE; 2016.

18. Fundação Sistema Estadual de Análise de Dados (SEADE). Índice Paulista da Primeira Infância. [homepage na Internet] São Paulo. [acessado 2016 Jun 15] http:// www.ippi.seade.gov.br

19. World Health Organization (WHO). Physical Status: The Use and Interpretation of Anthropometry. Geneva: WHO; 1995. (WHO Technical Report Series no. 854).

20. de Onis M, Onyango AW, Borghi E, Siyam A, Nishida C, Siekmann J. Development of a WHO growth reference for school-aged children and adolescents. Bull World Health Organ 2007; 85(9):660-667.

21. Machado-Júnior LC, Passini-Júnior R, Rodrigues-Machado I. Late prematurity: a systematic review. J Pediatr (Rio J) 2014; 90(3):221-231.

22. Kiy AM, Rugolo LMSS, De Luca AKC, Corrente JE. Growth of preterm low birth weight infants until 24 months corrected age: effect of maternal hypertension. J Pediatr (Rio J) 2015; 91(3):256-262

23. Amorim R, Carvalho-Lima M, Lira PI, Emond AM. Does low birthweight influence the nutritional status of children at school age? A cohort study in northeast Brazil. Matern Child Nutr 2011; 7(3):295-306.

24. Karlberg J, Albertsson-Wikland K. Growth in full-term small-for-gestational-age infants: from birth to final height. Pediatr Res 1995; 38(5):733-739.

25. Souza VCB, Hirakata V, Goldani MZ, Silva CH. Temporal evolution of the risk factors associated with low birth weight rates in Brazilian capitals (1996-2011). Population Health Metrics 2016; 14(15):1-10.

26. Addo OY, Stein AD, Fall CH, Gigante DP, Guntupalli AM, Horta BL, Kuzawa CW, Lee N, Norris SA, Prabhakaran P, Richter LM, Sachdev HS, Martorell R; Consortium on Health Orientated Research in Transitional Societies (COHORTS) Group. Maternal height and child growth patterns. J Pediatr 2013; 163(2):549-554.

27. Villar J, Altman DG, Purwar M, Noble JA, Knight HE, Ruyan P, Cheikh Ismail L, Barros FC, Lambert A, Papageorghiou AT, Carvalho M, Jaffer YA, Bertino E, Gravett MG, Bhutta ZA, Kennedy SH; International Fetal and Newborn Growth Consortium for the 21st Century. The objectives, design and implementation of the INTERGROWTH-21 $1^{\text {st }}$ Project. BJOG 2013; 120(Supl. 2):S9-S26. 
28. Crozier SR, Inskip HM, Godfrey KM, Cooper C, Harvey NC, Cole ZA, Robinson SM; Southampton Women's Survey Study Group. Weight gain in pregnancy and childhood body composition: findings from the Southampton Women's Survey. Am J Clin Nutr 2010; 91(6):1745-1751.

29. Euser AM, de Wit CC, Fiken MJ, Rijken M, Wit JM. Growth of preterm born children. Horm Res 2008; 70(6):319-328.

30. Leger J, Limoni C, Collin D, Czernichow P. Prediction factors in the determination of final height in subjects born small for gestational age. Pediatr Res 1998; 43(6):808-812.

31. Jaquet D, Deghmoun S, Chevenne D, Collin D, Czernichow P, Lévy-Marchal C. Dynamic change in adiposity from fetal to postnatal life is involved in the metabolic syndrome associated with reduced fetal growth. Diabetologia 2005; 48(5):849-855.

32. Eriksson JG, Forsén T, Tuomilehto J, Osmond C, Barker DJ. Early growth and coronary heart disease in later life: longitudinal study. BMJ 2001; 322(7292):949-953.

33. Eriksson J, Forsén T, Tuomilehto J, Osmond C, Barker D. Size at birth, childhood growth and obesity in adult life. Int J Obes Relat Metab Disord 2001; 25(5):735-740.

34. Huang RC, Mori TA, Burrows S, Le Ha C, Oddy WH, Herbison C, Hands BH, Beilin LJ. Sex dimorphism in the relation between early adiposity and cardiometabolic risk in adolescents. J Clin Endocrinol Metab 2012; 97:E1014-1022

Artigo apresentado em 28/07/2016

Aprovado em 26/01/2017

Versão final apresentada em 28/01/2017 